\title{
Perspective
}

\section{COVID-19 Waves: Importance of Accumulative Mortality per Million Inhabitants}

\author{
Carlos A. Delgado, ${ }^{1,2}$ Roberto L. Shimabuku ${ }^{1}$ and Pamela M. Chiroque-Solano ${ }^{3}$ \\ ${ }^{1}$ Facultad de Medicina, Universidad Nacional Mayor de San Marcos, Lima, Perú \\ ${ }^{2}$ Instituto Nacional de Salud del Niño, Lima, Perú \\ ${ }^{3}$ Universidade Federal do Rio de Janeiro, Rio de Janeiro, Brazil
}

\begin{abstract}
The reported number of new cases underestimates the real spread of COVID-19 pandemic because of non-tested asymptomatic people and limited global access to reliable diagnostic tests. In this context, COVID-19 mortality with confirmed diagnosis becomes an attractive source of information to be included in the analysis of perspectives and proposals. Objective data are required to calculate the capacity of resources provided by health systems. New strategies are needed to stabilize or minimize the mortality surge. However, we will not afford this goal until more alternatives were available. We still need an effective treatment, an affordable vaccine, or a collective achievement of sufficient immunity (reaching up to $70 \%$ of the whole population). At any time, the arriving waves of the pandemic are testing the capacity of governments. The health services struggle to keep the plateau in a steady-state below 100 deaths per million inhabitants. Therefore, it is necessary to increase the alternatives and supplies based on the current and near-future expected demands imposed by the number of deaths by COVID-19. Estimating COVID-19 mortality in various scenarios with the gradual release of social constraints will help predict the magnitude of those arriving waves.
\end{abstract}

Keywords: COVID-19; mortality; pandemics; public health; registries

Tohoku J. Exp. Med., 2020 May, 251 (1), 47-49.

\section{Perspective}

The use of interactive maps and information systems allow real-time monitoring of the progress of the COVID19 pandemic in the world (Kamel Boulos and Geraghty 2020). Despite this, global access to diagnostic tests limits the actual assessment of the problem because the number of new cases underestimates the real value of incident cases, and under-registration has variable magnitude. As about $80 \%$ of those affected show no symptoms, it is also clear that there will be under-registration of the number of cases recovered. In this context, COVID-19 mortality with confirmed diagnosis becomes a unique source of information to be included in the analysis of perspectives and proposal approach.

Social distancing imposed through social control measures reduces the number of COVID-19 contagions and has strong scientific evidence in its favor (Lewnard and Lo
2020). However, the pandemic will continue until the implementation of effective treatment, the global distribution of a protective vaccine that does not currently exist, or in the unlikely event of a favorable virus mutation. In the meantime, many countries implemented strategies such as Mitigation and Suppression. The mitigation of progressive course seeks to stop the spread of the pandemic. Suppression reverses epidemic growth by reducing the number of cases to low levels and keeping that situation indefinitely (Ferguson et al. 2020). Mitigation in Peru, with social distancing and selective isolation, can "flatten the curve" and give time to health services. However, any measure can generate significant regrowth by releasing mitigation or suppression. It is difficult to know what the most appropriate strategy is. We also do not know how much time we have or how many times the "waves" of the disease will lash out over health services, at the end of quarantine. In every country, unexpected requirements must exceed

Received April 20, 2020; revised and accepted May 11, 2020. Published online May 28, 2020; doi: 10.1620/tjem.251.47.

Correspondence: Roberto L. Shimabuku, M.D., Ph.D., Facultad de Medicina, Universidad Nacional Mayor de San Marcos, Av. Grau 700, Lima 1, Perú.

e-mail: rshimabukua@unmsm.edu.pe

(C)2020 Tohoku University Medical Press. This is an open-access article distributed under the terms of the Creative Commons Attribution-NonCommercial-NoDerivatives 4.0 International License (CC-BY-NC-ND 4.0). Anyone may download, reuse, copy, reprint, or distribute the article without modifications or adaptations for non-profit purposes if they cite the original authors and source properly.

https://creativecommons.org/licenses/by-nc-nd/4.0/ 
health services supplies at any time.

In several countries and in South America, social control measures implemented since March or April 2020 appear to be yielding results by progressively reducing mortality. As shown in Fig. 1, some countries like Spain, Sweden and the United States reach more than 100 deaths per million inhabitants, while others like Korea, New Zealand and Japan are below 10. However, not all countries see a trend of reaching a plateau soon, with minimal increase in deaths. The speed of response made a big difference in the first weeks, to avoid exceeding the capacity of health services. However, relaxing the control measures may trigger a new failure. At any time, the arriving waves of the pandemic are testing the capacity of the health services. It is therefore necessary to increase the alternatives and supplies based on the current demand imposed by the number of deaths by COVID-19. Estimating COVID-19 mortality in various scenarios with the gradual release of social constraints will help predict the magnitude of those arriving waves.

Fig. 1 shows mortality per million inhabitants in weeks since the first case in each country, and not by population density in affected areas. Avoiding density in the analysis could be a limitation because the affected areas by COVID-
19 do not always compromise all regions of each country. However, in the current context, a more realistic scenario should include the entire population as a denominator because there is no history of pre-COVID-19 immunity. It means that the entire population of any country is susceptible to contracting the disease at some point. Also, the accumulated mortality, shown in Fig. 1, might include causes other than COVID-19, overestimating the effect and affecting the arrival up to the maximum number of deaths. Hopefully, the adjustment measurements to keep around the plateau in a steady-state should achieve an aim below 100 deaths per million inhabitants.

The main advantage of representing weekly mortality per million inhabitants on a logarithmic scale is that it uses data not linked to the number of diagnosed (underestimated data). Besides, the mortality data is essential for trend analysis and mathematical models because it allows making inferences using more objectively data. Objective data are required to calculate the capacity of resources provided by health systems. We need to stabilize or minimize the mortality surge. However, we will not afford this goal, until more alternatives were available, such as collective immunity (reaching $70 \%$ or $80 \%$ of infected people in the population).

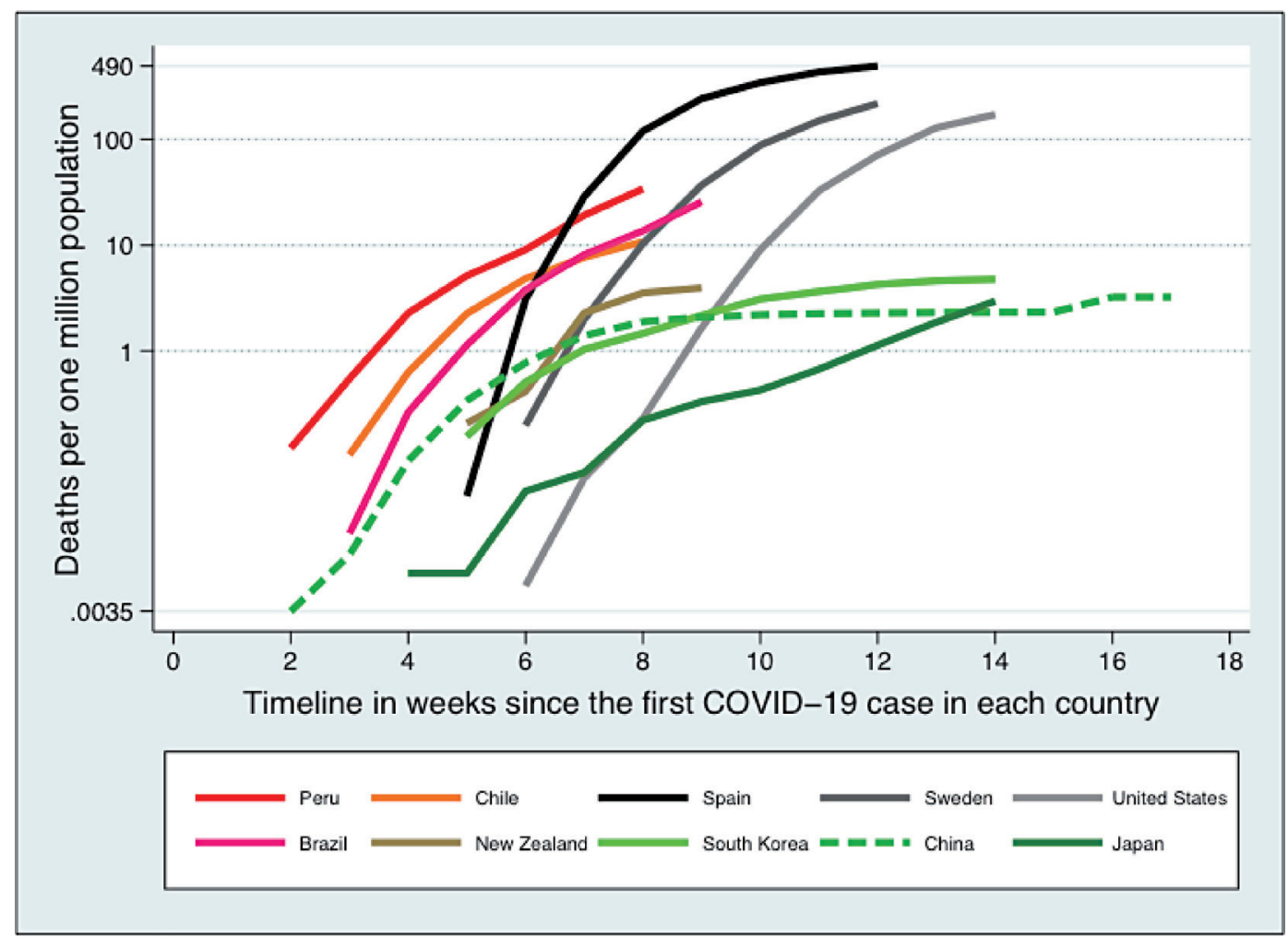

Fig. 1. Deaths per one million population in selected countries (Logarithmic scale).

Source: Information weekly updated until May 1st, 2020.

Population data: https://population.un.org/wpp/DataQuery/

China mortality: https://population.un.org/wpp/DataQuery/

Peru mortality: https://covid19.minsa.gob.pe/sala_situacional.asp

Mortality of other countries: https://coronavirus.app/map; https://coronavirus.jhu.edu/map.html

Graphic prepared by authors using Stata v16. 
Information on the COVID-19 mortality rate requires accurate definitions. COVID-19 lethality is the number of deaths among those diagnosed. However, the number of diagnosed does not reflect the actual number of infected. The real number of infected depends on the number of available tests, the sensitivity and specificity of those tests, and the evaluation coverage in the entire population. Although the number of diagnosed people underestimated the real value, it still is being used to display maps of information that are updated daily. One of those maps (Johns Hopkins) has already stopped reporting recoveries (which depends on those diagnosed) because they do not consider it to be reliable data for now.

COVID-19 mortality per million inhabitants reflects a more reasonable scenario, as opposed to lethality or recovered people information (which depends on the number of diagnosed). The number of COVID-19 deaths is harder to misestimate, even in Peru. However, the overestimation of the number of COVID-19 deaths also occurs when it includes deceased persons without a confirmed diagnosis. On the other hand, the total population in 2020 for each country, if precisely estimated by the United Nations. Absolute and cumulative COVID-19 mortality data can help make more accurate estimates in the future. More precise mortality data must be accumulated over time. A complicating factor is that there are reports of recovered COVID-19 patients testing positive again. Therefore, a mathematical model outlined for these analyses can be the SIERMD model: Susceptible - Infectious - Exposed Recovered - Medically Symptomatic - Dead. The SIERD model has principles based on classic ideas of transmitting infectious hosts (I) to susceptible hosts (S), including information from the recovered (R) and exposed (E) population (Anderson and May 1979).

Estimates in any proposed model should be made similar to what Anastassopoulou et al. (2020) published recently. Their study, based on a mathematical modeling approach, has provided relatively accurate three-week forecasts. The research supported the use of data from mortal- ity and is one of the first studies to suggest that the actual number of infections in the total population is in the order of twenty times more than those reported. Also, those researchers state that the mortality rate in the total population must be approximately $0.15 \%$. The accuracy in those results is related to the use of more real data, i.e., without variation in reported mortality and simulating variations in the other model parameters.

We conclude that mortality data, despite its limitations, is required to calculate the additional needed demand for resources. Currently, the health systems struggle with scarcity to resolve health demands to minimize mortality and maximize well-being. Therefore, we need to look at death numbers to find the know-how for saving lives.

\section{Conflict of Interest}

The authors declare no conflict of interest.

\section{References}

Anastassopoulou, C., Russo, L., Tsakris, A. \& Siettos, C. (2020) Data-based analysis, modelling and forecasting of the COVID-19 outbreak. PLoS One, 15, e0230405.

Anderson, R.M. \& May, R.M. (1979) Population biology of infectious diseases: part I. Nature, 280, 361-367.

Ferguson, N., Laydon, D., Nedjati Gilani, G., Imai, N., Ainslie, K., Baguelin, M., Bhatia, S., Boonyasiri, A., Cucunuba Perez, Z., Cuomo-Dannenburg, G., Dighe, A., Dorigatti, I., Fu, H., Gaythorpe, K., Green, W., et al. (2020) Report 9: impact of non-pharmaceutical interventions (NPIs) to reduce COVID19 mortality and healthcare demand.

https://www.imperial.ac.uk/mrc-global-infectious-diseaseanalysis/covid-19/report-9-impact-of-npis-on-covid-19/ [Accessed: May 1, 2020].

Kamel Boulos, M.N. \& Geraghty, E.M. (2020) Geographical tracking and mapping of coronavirus disease COVID-19/ severe acute respiratory syndrome coronavirus 2 (SARSCoV-2) epidemic and associated events around the world: how 21 st century GIS technologies are supporting the global fight against outbreaks and epidemics. Int. J. Health Geogr., 19, 8.

Lewnard, J.A. \& Lo, N.C. (2020) Scientific and ethical basis for social-distancing interventions against COVID-19. Lancet Infect. Dis., doi: 10.1016/S1473-3099(20)30190-0. [Epub ahead of print]. 